\title{
Care-seeking as a proxy indicator of the mental health of elderly Brazilians
}

\author{
Luis F. Castro-de-Araujo, , ${ }^{1,2}$ (iD Daiane B. Machado,, ${ }^{2,3}$ Maurício L. Barreto ${ }^{2,4}$ \\ ${ }^{1}$ Department of Psychiatry, University of Melbourne, Austin Health, Heidelberg, Victoria, Australia. ${ }^{2}$ Centro de Integração de Dados e \\ Conhecimentos para Saúde (CIDACS), Salvador, BA, Brazil. ${ }^{3}$ Centre for Global Mental health (CGMH), London School of Hygiene and \\ Tropical Medicine, London, England. ${ }^{4}$ Instituto de Saúde Coletiva, Universidade Federal da Bahia (UFBA), Salvador, BA, Brazil.
}

\begin{abstract}
Objective: To assess a large set of metadata made public by the Brazilian Ministry of Health on older subjects who visited outpatient mental health services in Brazil from 2008 to 2012.

Methods: We extracted data from the Brazilian Unified Health System Information Technology Department (Departamento de Informática do Sistema Único de Saúde, DATASUS), then calculated rates of visits per population in each of the five regions of Brazil, using census data for each year. Finally, logistic regressions were performed with depressive disorders or dementias as dependent variables, controlled by age and year of visit, stratified by region.

Results: Mood disorders were the leading reason for visits to outpatient mental health services by older adults, followed by delusional disorders. The calculated rates were lower than the known prevalence of depressive disorders and dementias, but the regressions revealed typical patterns. Males were less likely to present with a depressive disorder, while older subjects were more likely to present with depression and dementia.

Conclusions: Publicly available data from DATASUS may not enable inferences about the prevalence of mental disorders in elders, but inferential analyses match what is known about these conditions. This approach is supplemental to other more common ones and is of special importance for policymakers and health system managers.
\end{abstract}

Keywords: Epidemiology; old age psychiatry; big data; dementia; depression

\section{Introduction}

Brazil has made the demographic transition and is becoming an older country. This phenomenon has had several consequences, most notably regarding limited funds to support the growing population of retirees. This transition impacts the health system, as such subjects will require specialized health services. ${ }^{1}$ Within the field of mental health, longevity will also bring longer morbidity. ${ }^{2}$ The elder population is susceptible to multimorbidity as chronic conditions increase with age, and with these the number and severity of mental disorders in this population increase as well.

The epidemiology of mental health in older adults is of great importance to the management of the health system, as it can potentially reduce costs and morbidity by adequately matching staff training to the needs of a certain population. Prevalences are usually calculated using more traditional approaches, such as surveys. This information on prevalence of mental disorders in the elderly $^{3}$ yields public datasets that correspond to individuated, but anonymized, information on individuals who sought outpatient services. These datasets are usually

Correspondence: Luis Fernando Silva Castro de Araujo, Rua Mundo, 121, 41745-715, Salvador, BA, Brazil.

E-mail: Idearaujo@unimelb.edu.au

Submitted Mar 25 2019, accepted Dec 27 2019, Epub Jun 012020. very large and have been seldom analyzed. This work will use one such dataset, from the Brazilian Unified Health System Information Technology Department (Departamento de Informática do Sistema Único de Saúde, DATA SUS), to examine the reasons why older adults seek outpatient mental health services in Brazil. However, it is important to first summarize how mental health is organized in the country.

Psychiatric care in Brazil began in 1852, when the first psychiatric hospital was opened. Its main objective was to remove people with mental health problems, the homeless, and indigents from the streets. ${ }^{4}$ In 1970, inspired by the Italian experience of deinstitutionalization, a psychiatric reform movement against this hospital-centric care model gained traction. ${ }^{5}$ However, mental health care in Brazil was only officially reformulated in 1978, when Psychosocial Care Centers (Centros de Atenção Psicossocial, CAPS) were created to provide community-based care. $^{5}$

Mental health policy was subsequently redefined by building a network of mental health care centers to replace the previous model centered on hospitalization, alongside a progressive reduction in the number of existing 
psychiatric beds. ${ }^{6}$ Currently, specialized mental health care services are provided at CAPS. ${ }^{7}$

CAPS were created to provide care to some of the patients leaving mental hospitals. They are expected to offer outpatient, multidisciplinary care, including counseling, social services, occupational therapy, and clinical psychiatry consultations. Patients can be seen in three modalities: intensive care, semi-intensive care, and nonintensive care. In CAPS terminology, intensive refers to the frequency of attendance, which can range from one day a week to daily visits in more severe and persistent mental disorders. ${ }^{8}$

Both the number of CAPS and their coverage in the Brazilian territory have grown, from 148 in 1998 to 1,620 in 2010 and from $0.21 / 100,000$ population to $0.66 /$ 100,000 in the same period, respectively. ${ }^{9}$ The geographic distribution of these services was initially unequal; the Southeast (the most developed region of the country) had more of these facilities than the other Brazilian regions until 2005, when inequalities were addressed by the government, resulting in a shift towards an improved distribution. ${ }^{7,9}$

CAPS are also the main entry points to mental health services within the context of the Brazilian Unified Health System (Sistema Único de Saúde [SUS]). Subjects encounter few barriers to access these services, and data from CAPS consultations can be considered a good representation of Brazilian outpatient care. Older adults with mental disorders are mainly managed at these centers. Each consultation generates an administrative report, which is filled electronically and forwarded to the Ministry of Health for accounting and management purposes. These are called high-complexity procedure authorizations (Autorização de Procedimentos de Alta Complexidade [APACs]). APACs are then anonymized and made public on the DATASUS website (www2. datasus.gov.br). These are primarily destined for administrative purposes, but after being made public they effectively represent metadata for each subject who sought any of the numerous outpatient services within the SUS. This metadata includes some demographic information, but also the main diagnosis the subject was given upon consultation.

Information on the prevalence of mental disorders among older adults in Brazil is very fragmented. It is usually reported from surveys and cohorts, which are the best approach to determine prevalences and incidences. However, these are limited in the sense of reach across distinct Brazilian regions. There is no nationwide approach to the patterns of diagnosis that generate visits to outpatient services in Brazil. This is where routine health records come in useful.

Routine health records can be used to gauge the health status of a given population. This has been previously attempted for the subset comprising CAPS visits for children with mental disorders and autism. ${ }^{10,11}$ Due to the relative lack of studies using routine health records in Brazil, we set out to extend these previous studies by analyzing older adults (age $>60$ years) who were seen at CAPS from 2008 to 2012 . We hypothesized that the rates of visits per population would partially mirror the known prevalence of mental disorders. Therefore, we plan to compare the rates found to those of other major surveys completed in Brazil. Finally, we plan to test the existence of some basic associations repeatedly found in this population, such as the relationships of gender and age with both depressive symptomatology and dementia. We hypothesize that such statistical tests will reflect what is known about these disorders, with a higher risk of depression among women and a higher risk of depression and dementia with advancing age.

\section{Methods}

We used data from outpatient services in Brazil to analyze the mental health care profile (number of visits and diagnosis) of people over age 60 who attended these services from 2008 to 2012 . Overall, $92 \%$ of the data $(1,037,435$ of $1,124,244)$ were from CAPS, and the remainder from other outpatient services.

\section{Data source}

All data were processed, anonymized, and made available by DATASUS (www2.datasus.gov.br). Data were extracted using the steps described below.

\section{Data extraction}

Information from APACs was extracted from the service area in the file transfer option. The files can be retrieved within the Outpatient Information System (SIASUS), under the name miscellaneous AD-APAC reports (ADAPAC de laudos diversos).

As all data had been previously anonymized and made public by the Brazilian Ministry of Health, there was no need for ethical approval.

Data management, import, and analyses were all performed in R, version 3.5.1 (https://cran.r-project.org/). After data import and subsetting for subjects older than 60 years, the final number of observations was 1,124,244.

\section{Inferential analyses}

Rates of visits by 100,000 inhabitants were calculated for each region. The Brazilian census is carried out by the Brazilian Institute of Geography and Statistics (IBGE) every 10 years. The most recent census was collected in 2010; data for later years are projections calculated by IBGE. Tables with rates of outpatient visits by 100,000 population (according to census data for the year) and stratified by Brazilian regions can be found in Table 1 for main ICD-10 blocks. Rates for each category within the block of depressive disorders and dementia are presented in the online-only supplementary material. In Table 1, we performed chi-square contingency table tests for the categorical variables and one-way analysis of variance (ANOVA) for continuous variables. Logistic regression models were used to test whether depressive disorders (any category within and including F32 and F33) or dementias (any category within and including F00-F03) were associated with sex, age, and year, stratified by regions. 


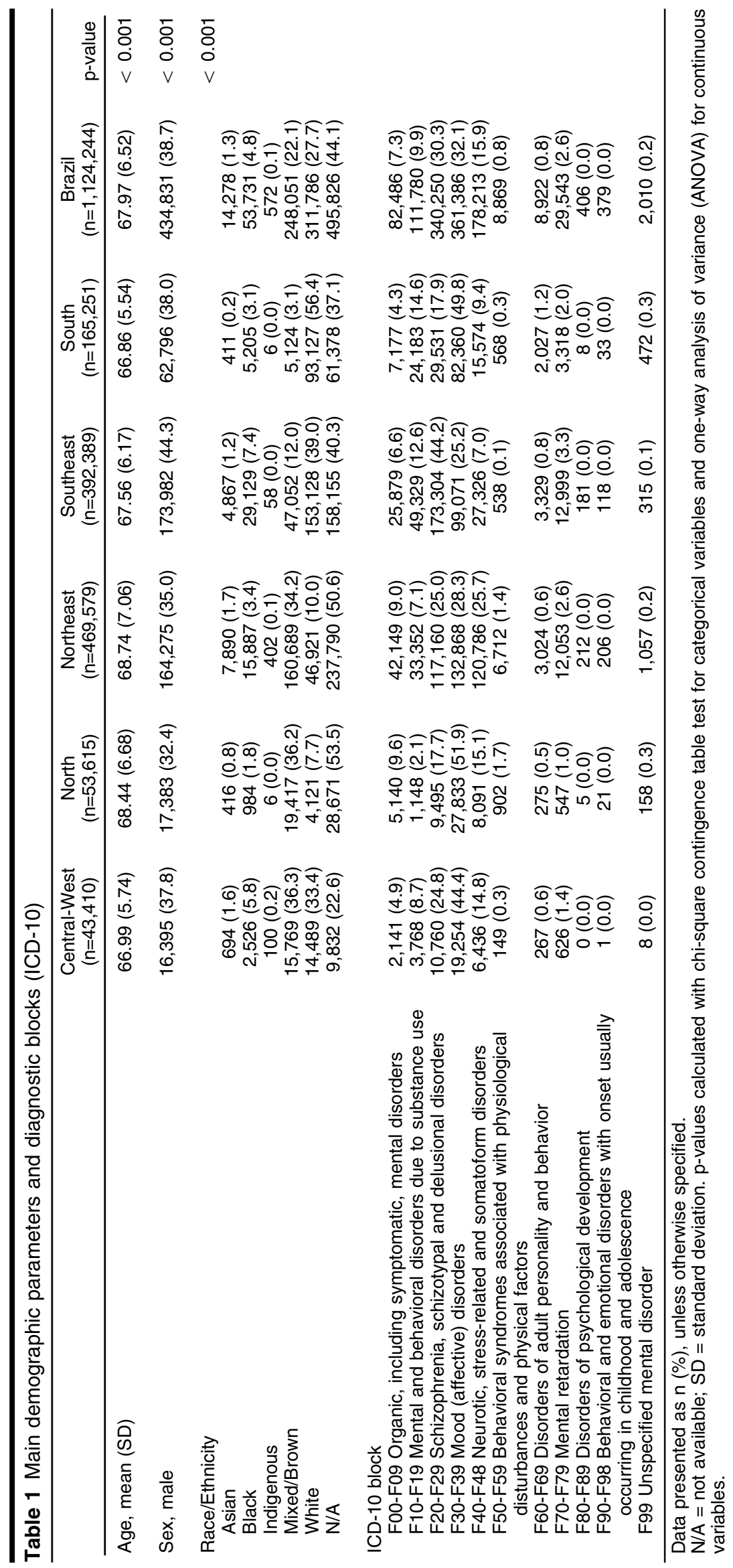


The age strata were cut into quartiles $(63-66,66-71$, and 71-99 years). Model estimates are provided in the onlineonly supplementary material. The year was included in the model as a way of controlling for the known yearly increase in the number of CAPS in Brazil.

\section{Results}

Main descriptive statistics can be found in Table 1, as well as a boxplot for the age in Figure S1, available as online-only supplementary material. Data on substance use-related disorders, which comes from a specialized subtype of CAPS, was also used to generate Figure 1. The proportion of disorders was calculated by Brazilian region. Women made more visits to CAPS overall (Table 1, Figure S2, Figure 2, and Figure 3).

Starting by the Central-West region (Table 1), we found that the leading block of conditions that led to CAPS visits was mood disorders (F30-F39, 44.4\%), followed by schizophrenia, schizotypal, and delusional disorders (F20-F29, $24.8 \%)$. The North region followed a similar pattern, with $51.9 \%$ and $17.7 \%$ respectively. The Northeast region had a different pattern: mood disorders (F30-F39, 28.3\%) remained the most common type of disorders, followed by neurotic, stress-related, and somatoform disorders (F40-F48, 25.7\%).

In Figure 1 the rate of visits per 100,000 population according to census data for each of the studied years is shown. Some findings are of particular interest. The Northeast region has the highest levels of neurotic, stress-related, and somatoform disorders across all regions, reaching $500 / 100,000$ in 2011 and 2012. The Northeast and Southeast presented similar rates of visits due to delusional disorders. The South region had the highest rates of visits due to mood disorders, exceeding 600/100,000 in 2011.

The geographic pattern for the year 2012 can be seen in Figure 1. The North had the highest rate of visits for depressive disorders (F32-F33), while the Northeast had the highest rates of visits due to dementia (F00-F03). Trends from 2008 to 2012 are shown in Figure 1.

Within the block of organic disorders, the category that drove the most visits to outpatient mental health services (Table S1, available as online-only supplementary material) was dementia in Alzheimer disease with early onset (F00.0), followed by dementia in Alzheimer disease with late onset (F00.1). There was a striking regional difference in this finding, with the Northeast having the highest rate of both conditions compared to the other regions.

We performed logistic regressions with depressive disorders as the dependent variable. A full report of the estimates can be found in Table S2 (online-only supplementary material), alongside Nagelkerke's $R^{2}$ for each model. Figure 2 summarizes our findings using average marginal effects. It shows that being male is a protective effect in relation to visiting an outpatient mental health facility for depressive disorder. This effect varies across regions. In the South, for example, each unit increase in the categorial dependent variable (presence of depressive disorder) led to a decrease of 0.19 units in the
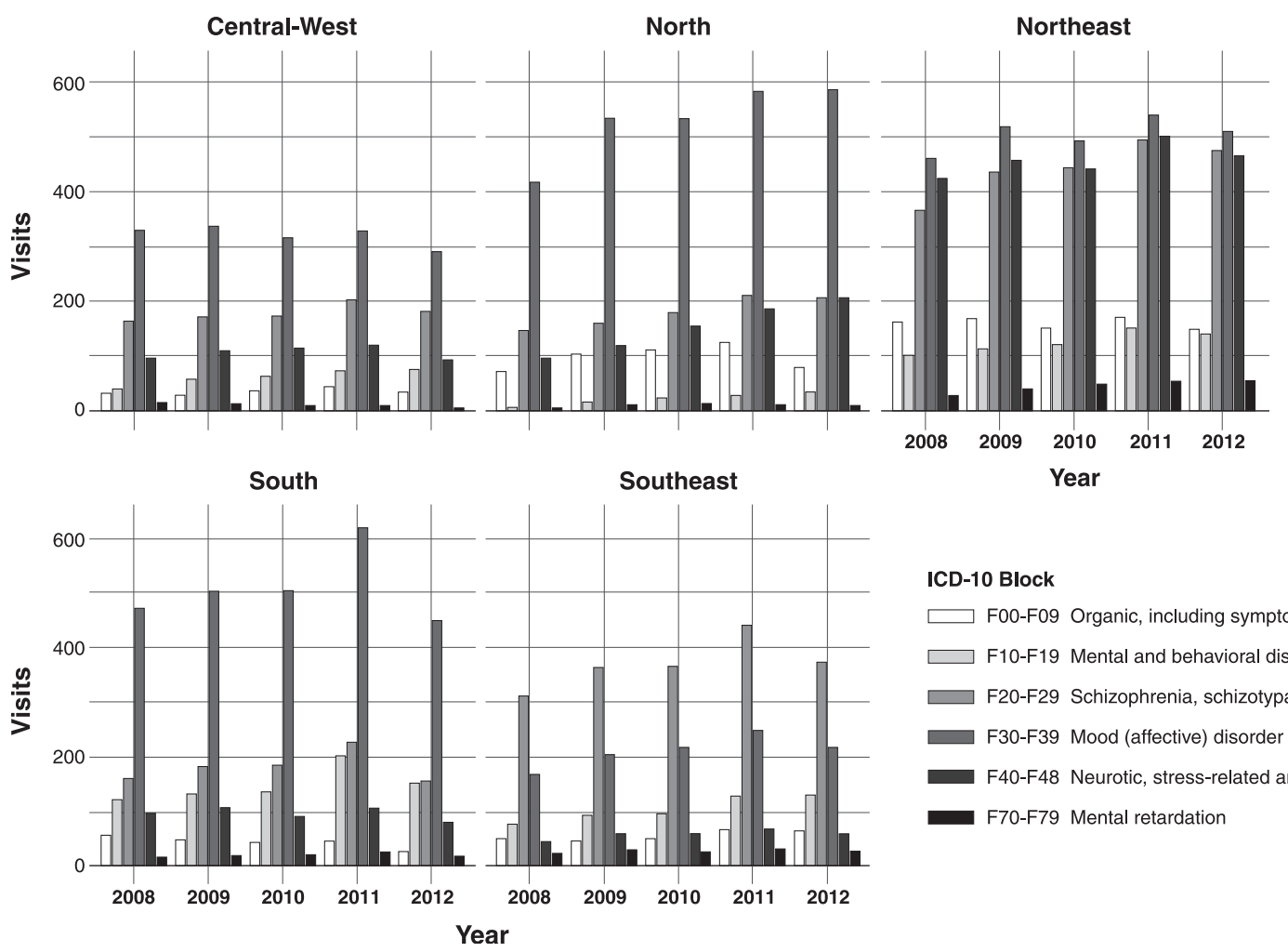

ICD-10 Block

$\square$ F00-F09 Organic, including symptomatic, mental disorder

$\square$ F10-F19 Mental and behavioral disorder due to substance use

$\square$ F20-F29 Schizophrenia, schizotypal and delusional disorder

F30-F39 Mood (affective) disorder

F40-F48 Neurotic, stress-related and somatoform disorder

F70-F79 Mental retardation

Figure 1 Visits to outpatient mental health services per 100,000 population (according to census estimates for the corresponding year), stratified by mental disorders as ICD-10 blocks. 

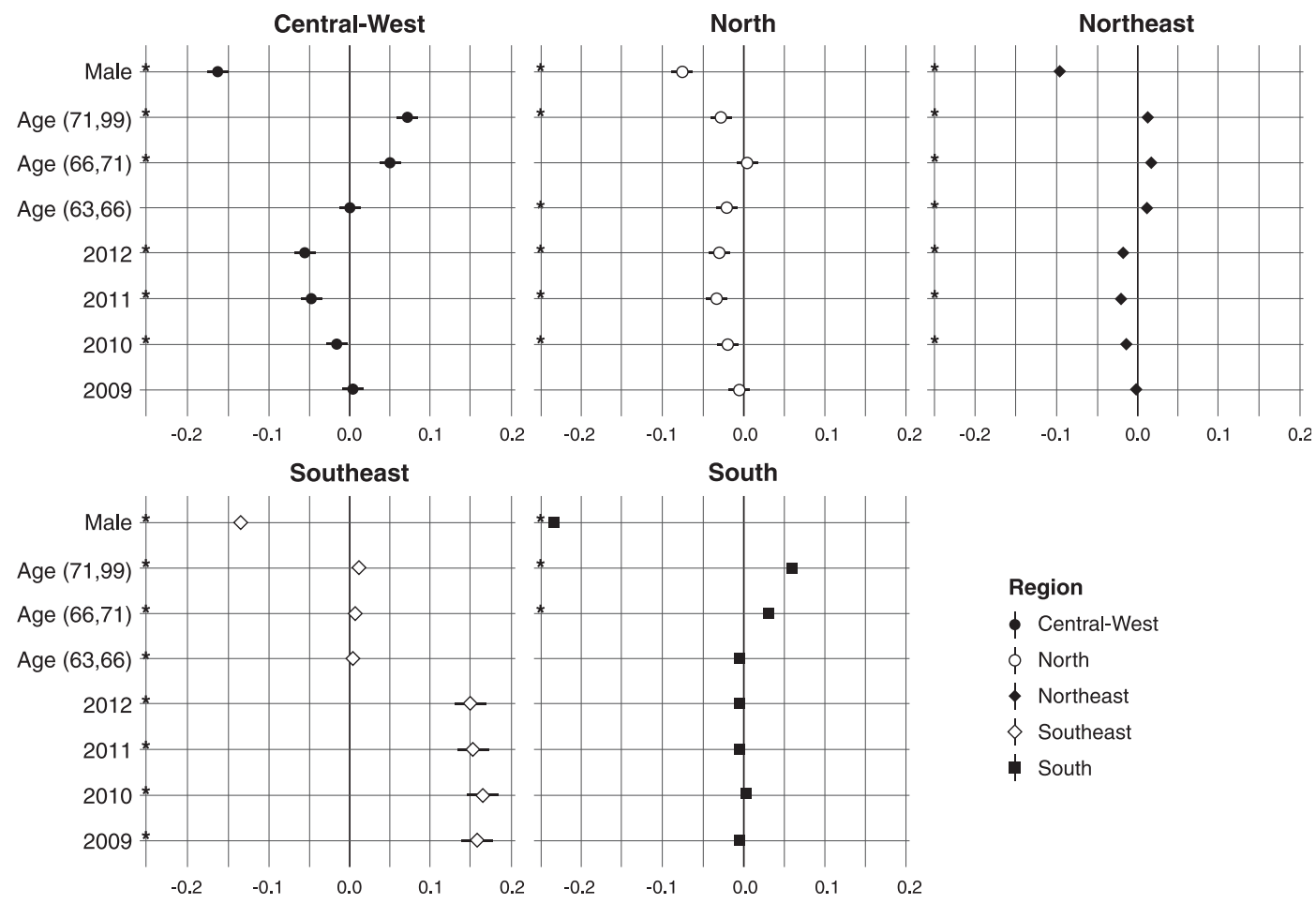

Average marginal effect

Figure 2 Average marginal effects of the regression model for depressive disorders. $* p<0.05$.
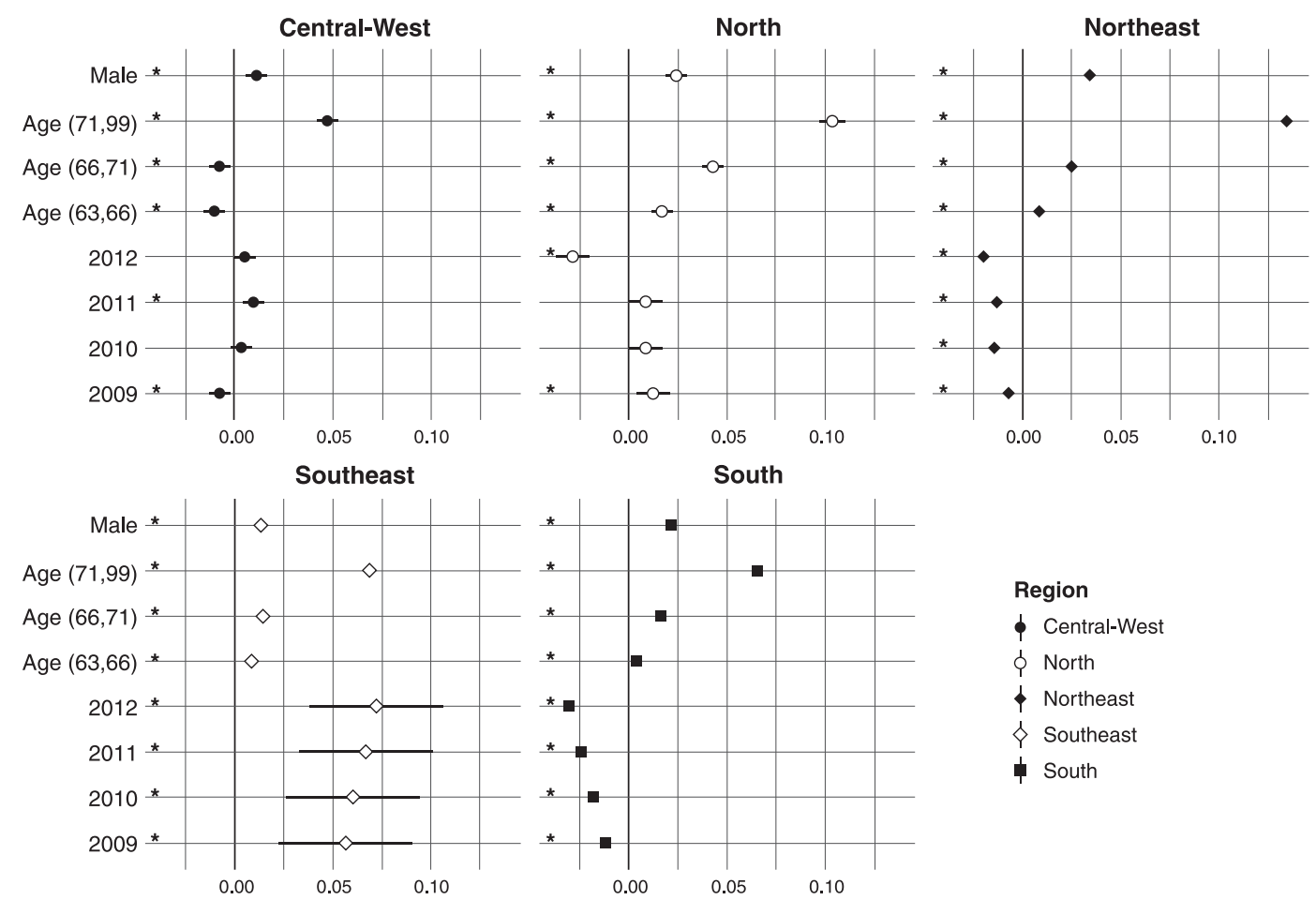

Region

- Central-West

o North

- Northeast

$\diamond$ Southeast

South

Average marginal effect

Figure 3 Average marginal effects of the regression model for dementias. ${ }^{*} p<0.05$. 
dependent variable male sex. This can be read as a probability as well; hence, a $19 \%$ decrease (Figure 2). All Nagelkerke's $\mathrm{R}^{2}$ values were very small, but this was expected. Regarding age ranges, there was a trend for older subjects to be at higher risk of attending an outpatient service with a depressive disorder, except in the North and Northeast regions, where the very old did not have a higher risk of seeking care for a depressive disorder.

Similar logistic regressions were performed with dementias as the dependent variable. A full report of the estimates can be found in Table S3 (online-only supplementary material), again with Nagelkerke's $R^{2}$ for each model. Figure 3 summarizes our findings with marginal effects. In this case, we found that males were at higher risk. In the Northeast, for instance, males were 3\% more likely to attend for each unit rise of the dependent variable. On the other hand, age had a much clearer relationship with dementias, with the very old (71-99 years) having the highest risk. Again, Nagelkerke's $\mathrm{R}^{2}$ were very small, which was expected, as these models can only represent a small part of the elements that are related to the outcome (a mental disorder, which are typically very complex conditions).

\section{Comparison to existing data}

The rate of care-seeking for all types of depressive conditions (F32-F33) (Table S1) amounted to 1,573.85/ 100,000 , or $15 \%$. On the other hand, previous reports of the prevalence of depression in older adults in Brazil ranged from 4.7 to $36.8 \%$. $^{3}$ The same calculation for all dementias in ICD-10 gives 232.51 , or $2.32 \%$, while the calculated prevalence of dementia in Brazil ranges from 4.3 to $29 \%$ according to Blay et al. ${ }^{3}$ Rates are within previously calculated prevalence ranges.

\section{Discussion}

This study represents an attempt to examine the epidemiology of psychiatric conditions in Brazilian older adults through government-provided metadata. This information is originally collected for administrative purposes, but is anonymized and made available online for the public to inspect, which makes it an attractive data source for research. Extraction of this information for the entire country requires computational power that has become available only relatively recently. We extracted the data, created a subset restricted to older adults, calculated rates to allow comparison across different regions, and performed two inferential tests to ascertain whether the data reflects what is known of two mental disorders that are of particular importance to geriatric psychiatry: dementias and depressive disorders. We found important regional differences with regard to rates of visits due to these and other mental health conditions. The highest rates of visits for depressive disorders were found in the South, and were substantial in the Northeast as well. Dementias are leading causes of visits to outpatient services in the Northeast region. Anxiety disorders accounted for a high proportion of visits in the Northeast, while delusional disorders were most common in the Northeast and Southeast. Upon running a formal inferential test with depressive disorders and dementias as outcomes, we found that females were at higher risk of depression and that older subjects have a much higher risk of dementia. As a result, the findings of this study seem to provide an interesting, though partial, picture of the mental health of elderly Brazilians.

The rate of visits per population facilitates comparisons between regions. Indeed, examination of the various diagnoses across regions reveals several interesting differences. Inspection of the geographic distribution of depressive conditions and dementias in 2012 (Figure 4) reveals stark regional differences. The North had higher rates of visits due to depressive conditions (within categories F32-F33). Trends for each of the studied years can be found in Figure 1. Visits to outpatient services for mood disorders were highest in the South $(>600 / 100,000$ in 2011) and in the North (> 500/100,000 in 2009, 2010, 2011, and 2012). On the other hand, delusional disorders (F20-F29) rates were highest in the Southeast, reaching $400 / 100,000$ in 2011. The depressive categories with the highest rates were, in order: Mild depressive episode (F32.0), Moderate depressive episode (F32.1), Recurrent depressive disorder, current episode moderate (F33.1), and Recurrent depressive disorder, current episode mild (F33.0) (Table S1).

Previous work has shown that the prevalence of depression in older adults attending outpatient care in Brazil is $36 \%{ }^{12}$ or higher for clinically significant depressive symptoms (39\%). Depressive disorders in the elderly are known to be comorbid with anxiety and alcohol dependence. $^{2}$ In this work, we found mood disorders to be the main overall motivator for visits to outpatient services (Figure 5). Mild depressive episodes accounted for the highest rates of visits per population (413.24/ 100,000), followed by moderate episodes (300.21/ $100,000)$ and recurrent depressive disorder with current moderate episode $(183.41 / 100,000)$ (Table S1). There were striking regional differences, however. Visitation rates for mild depressive episode varied from 30.56/ 100,000 in the Southeast to $145.84 / 100,000$ in the North. For a recurrent depressive episode with a current mild episode, the South had the highest rates $(57.94 / 100,000)$, followed by the Northeast region with 42.05/100,000.

Statistical tests showed that females were more likely to visit CAPS due to depression. The Central-West, Southeast, and South regions followed the expected pattern, i.e., the older the subject, the higher the risk of depressive disorder. In the Northeast region, age did not change the risk, and in the North there was actually a reduction in the last (71-99) age strata Figure 2.

The prevalence of dementia in Brazil varies from $7.1 \%{ }^{3}$ to $17.5 \%,{ }^{13}$ while that of cognitive impairment can vary from 4.3 to $29.7 \%$. $^{3}$ Assessment of cognitive impairment depends on the subject's level of education, which is arguably low among Brazilian older adults; this might have impacted the prevalence reported by Blay et al. ${ }^{3}$ The present study found that the Northeast region of the country had the highest rates of CAPS visits due to organic mental disorders. When analyzing the distinct categories, we found that dementias were the culprit, leading to more visits in the Northeast than elsewhere in 
A

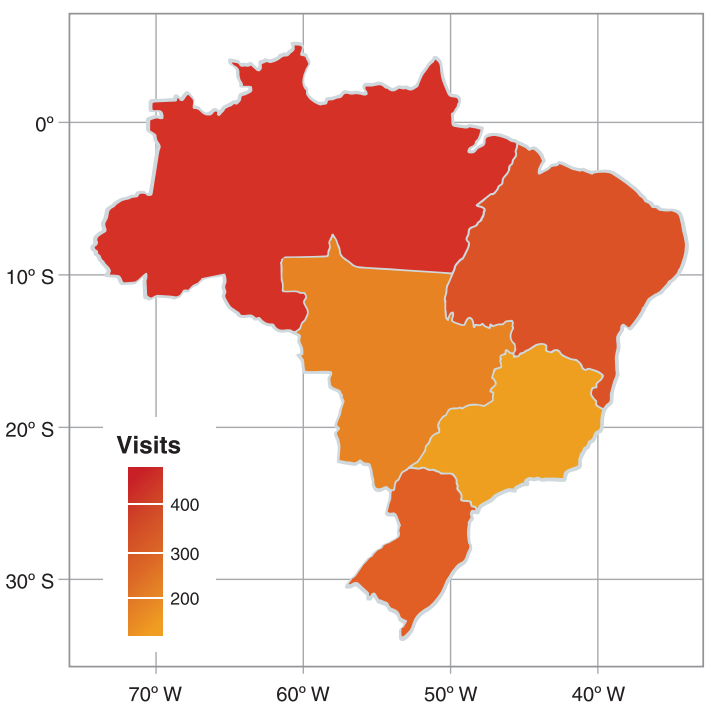

B

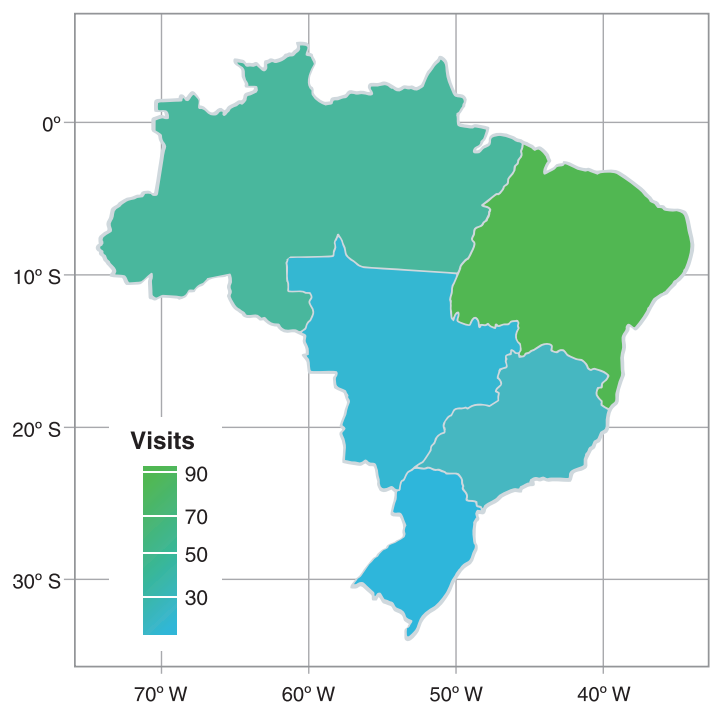

Figure 4 Geographic differences in rates for visits due to A) depressive disorders (ICD-10 F32.0-F33.9) and B) dementias (F00.0-F03) in 2012. Rates are calculated per 100,000 population using census projections for 2012.

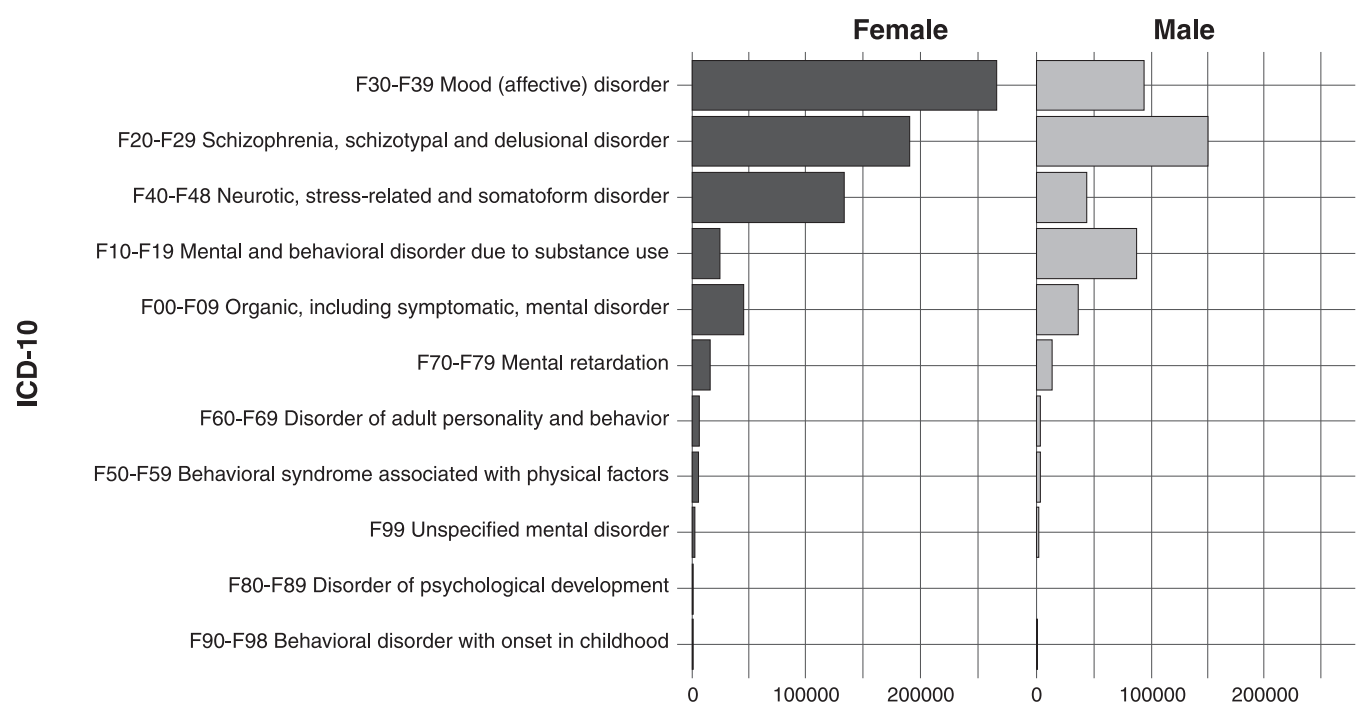

Figure 5 Most common conditions that resulted in a visit to outpatient mental health services. Absolute number of visits.

2012 (Figure 4). Within the dementias, the conditions that accounted for the highest rates overall were dementia in Alzheimer disease with early onset $(60.53 / 100,000)$, followed by dementia in Alzheimer disease with late onset $(22.96 / 100,000)$. The Northeast region presented a similar pattern, with rates of 22.85 and $11.15 / 100,000$ respectively.

A regression was performed with dementias (F00 to F03) as outcomes, which revealed that males had the highest probability of presenting to outpatient services with this diagnosis, as can be seen in Figure 3. The highest probability was found in the Northeast region, with males presenting an average marginal effect of 0.026 , or a $2.6 \%$ increase in probability for each unit increase in the outcome (a diagnosis of dementia). Also, as expected, age was consistently related to the outcome, with the oldest quartile presenting the highest probability. There has never been a report of higher levels of dementia in the Northeast compared to other regions in the country. We consider this attributable to access to mental health services, as CAPS are culturally not seen as services that offer care to the elderly. Therefore, this finding might be due to supply or demand factors mediating access to these services by this population.

There were some problems with the quality of the available data, mostly related to missing values. We found high levels ( $>50 \%$ ) of missing data for several variables, including secondary ICD-10 code, associated ICD-10 
code, and race. We did not use any of these variables in our analyses, but we reported race in Table 1 , as we found it to still be informative with regard to the ethnic distribution of the Brazilian population. Since in this data it was impossible to detect whether the APAC was for a returning patient or for a new subject, we expect that the information is inflated (although rates were ultimately much smaller than previously reported prevalences). This has consequences for the logistic regressions as well, as the model assumes linearity and absence of interactions that certainly exist in this dataset. Therefore, conclusions are not meant to be causal, but should only be seen as reflecting trends in mental health service attendance in Brazil.

In conclusion, public-domain data provided by DATASUS is equivalent to metadata on subjects who seek mental health services. As the Brazilian universal health system offers easy access to consultations, we believe our findings are representative of actual visits to these services nationwide. We were interested in whether analyses using these metadata would reflect what is found elsewhere by surveys and other studies. Our study shows that this very large data set does not perfectly mirror known prevalences of mental disorders in Brazil. Diseases such as schizophrenia require regular visits to outpatient services, which inflates their presence in the analyses; thus, we performed tests on depression and on dementias. For these conditions, we still found that rates are lower than calculated prevalences, which we find is due to supply and demand factors that could not be controlled due to the characteristics of this dataset. Nevertheless, the distributions of visits across the regions partially reflect what is expected of the conditions, and the relationships regarding age and gender are significant and reproduce expected patterns. Our findings complement those of surveys - in particular, by revealing distributions of visits, which can surely help decisionmakers match staff training to the particular needs of each region.

\section{Acknowledgements}

LFCA and DBM are funded by The Wellcome Trust via a research associate scholarship at Centro de Integração de Dados e Conhecimentos para a Saúde (CIDACS), Fundação Oswaldo Cruz (Fiocruz).

\section{Disclosure}

The authors report no conflicts of interest.

\section{References}

1 Dieleman JL, Squires E, Bui AL, Campbell M, Chapin A, Hamavid H, et al. Factors associated with increases in US health care spending, 1996-2013. JAMA. 2017;318:1668-78.

2 Blazer DG, Steffens DC. Depressive disorders. In: Steffens DC, Blazer DG, Thakur ME, editors. The American psychiatric publishing textbook of geriatric psychiatry. Washington: American Psychiatric Publishing; 2015.

3 Blay S, Laks J, Nitrini R, Caramelli P. Epidemiologia dos transtornos mentais em idosos e a utilização dos serviços por esta população. In: Mello MF de, Mello A de AF de, Kohn R, editores. Epidemiologia da saúde mental no Brasil. São Paulo: Artmed; 2007.

4 Oda AMGR, Dalgalarrondo P. História das primeiras instituições para alienados no Brasil. Hist Cienc Saude-Manguinhos. 2005;12:9831010.

5 Brasil, Ministério da Saúde, Secretaria de Atenção a Saúde. Reforma psiquiátrica e política de saúde mental no Brasil. Documento apresentado à Conferência Regional de Reforma dos Serviços de Saúde Mental: 15 anos depois de Caracas. Brasília: OPAS; 2005.

6 Mello MF de, Mello A de AF de, Kohn R. Epidemiologia da saúde mental no Brasil. São Paulo: Artmed; 2007.

7 Delgado PGG, Schechtman A, Ewber R, Amstalden AF, Bonavigo E, Cordeiro $F$, et al. Reforma psiquiátrica e política de saúde mental no Brasil. In: Mello MF, Mello AAF, Kohn R, editores. Epidemiologia da saúde mental no Brasil. São Paulo: Artmed; 2007. p. 39-84.

8 Pitta AM. [An Assessment of Brazilian Psychiatric Reform: Institutions, Actors and Policies]. Cien Saude Colet. 2011;16:4579-89.

9 Brasil, Ministério da Saúde. Saúde mental em dados 8 [Internet]. 2011 Jan [cited 2020 Feb 27]. bvsms.saude.gov.br/bvs/periodicos/ saude_mental_dados_v8.pdf

10 Ceballos GY, Paula CS, Ribeiro EL, Santos DN. Child and adolescent psychosocial care center service use profile in Brazil: 2008 to 2012. Braz J Psychiatry. 2019;41:138-47.

11 Garcia GY, Santos DN, Machado DB. [Psychosocial care centers for children and adolescents in Brazil: geographic distribution and user profile]. Cad Saude Publica. 2015;31:2649-54.

12 Castro-de-Araujo LF, Barcelos-Ferreira R, Martins CB, Bottino CM Depressive morbidity among elderly individuals who are hospitalized, reside at long-term care facilities, and are under outpatient care in Brazil: a meta-analysis. Braz J Psychiatry. 2013;35:201-7.

13 César KG, Brucki SM, Takada LT, Nascimento LF, Gomes CM, Almeida MC, et al. Prevalence of cognitive impairment without dementia and dementia in Tremembé, Brazil. Alzheimer Dis Assoc Disord. 2016;30:264-71. 